Supporting Information

\title{
Particle size effects on gas transport properties of 6FDA-Durene/ZIF-71 mixed matrix membranes
}

\author{
Susilo Japip $¥$ Youchang Xiao ${ }^{\ddagger}, \perp$, Tai-Shung Chung ${ }^{\ddagger} *$ \\ ${ }^{\ddagger}$ Department of Chemical and Biomolecular Engineering, \\ National University of Singapore, Singapore 117585 \\ ${ }^{\perp}$ Suzhou Faith \& Hope Membrane Technology Ltd Co., SIP, Jiangsu, PR China
}

*Corresponding author

Email: chencts@,nus.edu.sg

Fax : (65)-67791936 
Gas transport properties of the fabricated membranes and ZIF-71 estimated from Thornton et al. $^{1}$

Table S1. Pure gas permeability of the pristine 6FDA-Durene and 6FDA-Durene-ZIF71 MMMs with different particle sizes at $35^{\circ} \mathrm{C}$ and their corresponding ZIF-71 loadings.

\begin{tabular}{lcccccc}
\hline \multirow{2}{*}{ Membranes } & ZIF-71 & \multicolumn{5}{c}{ Pure Gas Permeability (Barrer) } \\
\cline { 3 - 7 } & Loadings (\%) & $\mathbf{H}_{\mathbf{2}}$ & $\mathbf{O}_{\mathbf{2}}$ & $\mathbf{N}_{\mathbf{2}}$ & $\mathbf{C O}_{2}$ & $\mathbf{C H}_{4}$ \\
\hline 6FDA-Durene & 0 & $760 \pm$ & $184 \pm$ & $55.0 \pm$ & $805 \pm$ & $47.6 \pm$ \\
6FDA-Durene- & & 30 & 10 & 5.2 & 12 & 4.8 \\
ZIF71-30nm & $21.2 \pm 2.4$ & $2099 \pm$ & $575 \pm$ & $186 \pm$ & $2560 \pm$ & $181 \pm$ \\
6FDA-Durene- & & 145 & 47 & 20 & 159 & 21 \\
ZIF71-200nm & $21.1 \pm 0.7$ & $2470 \pm$ & $643 \pm$ & $207 \pm$ & $2744 \pm$ & $198 \pm$ \\
6FDA-Durene- & & 110 & 33 & 13 & 114 & 13 \\
ZIF71-600nm & $19.3 \pm 1.1$ & $1526 \pm$ & $386 \pm$ & $120 \pm$ & $1656 \pm$ & $109 \pm$ \\
\hline
\end{tabular}

Table S2. Ideal gas selectivity of the pristine 6FDA-Durene and 6FDA-Durene-ZIF71 MMMs with different particle sizes at $35^{\circ} \mathrm{C}$ and their corresponding ZIF-71 loadings.

\begin{tabular}{lcccccccc}
\hline \multirow{2}{*}{ Membranes } & \multirow{2}{*}{$\begin{array}{l}\text { ZIF-71 } \\
\text { Loadings (\%) }\end{array}$} & \multicolumn{6}{c}{ Ideal Selectivity } \\
\cline { 3 - 8 } & $\mathrm{H}_{2} / \mathbf{C O}_{2}$ & $\mathbf{H}_{2} / \mathrm{N}_{2}$ & $\mathbf{H}_{2} / \mathbf{C H}_{4}$ & $\mathbf{O}_{2} / \mathbf{N}_{2}$ & $\mathbf{C O}_{2} / \mathbf{N}_{2}$ & $\mathbf{C O}_{2} / \mathbf{C H}_{4}$ \\
\hline 6FDA-Durene & 0 & $0.94 \pm$ & $13.9 \pm$ & $16.0 \pm$ & $3.35 \pm$ & $14.7 \pm$ & $17.0 \pm$ \\
& & 0.02 & 0.8 & 1.2 & 0.15 & 1.2 & 1.6 \\
6FDA-Durene- & $21.2 \pm 2.4$ & $0.82 \pm$ & $11.3 \pm$ & $11.6 \pm$ & $3.10 \pm$ & $13.8 \pm$ & $14.2 \pm$ \\
ZIF71-30nm & & 0.02 & 0.5 & 0.6 & 0.08 & 0.6 & 0.7 \\
6FDA-Durene- & $21.1 \pm 0.7$ & $0.90 \pm$ & $11.9 \pm$ & $12.5 \pm$ & $3.10 \pm$ & $13.2 \pm$ & $13.9 \pm$ \\
ZIF71-200nm & & 0.02 & 0.6 & 0.7 & 0.06 & 0.3 & 0.4 \\
6FDA-Durene- & $19.3 \pm 1.1$ & $0.92 \pm$ & $12.4 \pm$ & $13.5 \pm$ & $3.18 \pm$ & $13.5 \pm$ & $14.7 \pm$ \\
ZIF71-600nm & & 0.05 & 1.2 & 1.2 & 0.12 & 0.6 & 0.6 \\
\hline
\end{tabular}

Table S3. Gas transport properties of ZIF-71.

\begin{tabular}{cccccc}
\hline \multirow{2}{*}{ Sample } & \multicolumn{5}{c}{ Gas Permeability (Barrer) } \\
\cline { 2 - 6 } & $\mathrm{H}_{2}$ & $\mathrm{O}_{2}$ & $\mathbf{N}_{2}$ & $\mathbf{C O}_{2}$ & $\mathbf{C H}_{4}$ \\
\hline \multirow{4}{*}{ ZIF-71* } & 110,000 & 66,000 & 22,000 & 55,000 & 50,000 \\
\cline { 2 - 6 } & \multicolumn{5}{c}{ Ideal Selectivity } \\
\cline { 2 - 6 } & $\mathrm{H}_{2} / \mathbf{C O}_{2}$ & $\mathbf{H}_{2} / \mathbf{N}_{2}$ & $\mathbf{O}_{2} / \mathbf{N}_{2}$ & $\mathbf{C O}_{2} / \mathbf{N}_{2}$ & $\mathbf{C O}_{2} / \mathbf{C H}_{4}$ \\
\cline { 2 - 6 } & 2 & 5 & 3 & 2.5 & 1.1 \\
\hline *estimated from & Thornton et al $^{1}$ & &
\end{tabular}




\section{Reference}

(1) Thornton, A. W.; Dubbeldam, D.; Liu, M. S.; Ladewig, B. P.; Hill, A. J.; Hill, M. R. Feasibility of zeolitic imidazolate framework membranes for clean energy applications. Energy Environ. Sci. 2012, 5, (6), 7637-7646. 